\title{
Deficiency of hypoxia inducible factor-1 $\alpha$ promoted progression of diabetic nephropathy with hypertension
}

\author{
YUEJIANG JIAO ${ }^{1}$, HONGWEI JIANG ${ }^{2}$, HAIBO LU $^{2}$, YIPING YANG $^{1}$, \\ YANFANG ZHANG ${ }^{1}$, KUN ZHANG ${ }^{2}$ and $\mathrm{HUI} \mathrm{LIU}^{1}$ \\ ${ }^{1}$ Department of Endocrinology, Luoyang Central Hospital Affiliated to Zhengzhou University, Luoyang, Henan 471009; \\ ${ }^{2}$ Department of Endocrinology, The 1st Affiliated Hospital of Henan University of Science and Technology, \\ Luoyang, Henan 471033, P.R. China
}

Received October 9, 2017; Accepted July 13, 2018

DOI: $10.3892 / \mathrm{etm} .2018 .6621$

\begin{abstract}
The present study was designed to investigate the effect of hypoxia inducible factor- $1 \alpha(\mathrm{HIF}-1 \alpha)$ on diabetic nephropathy (DN) with hypertension. HIF-1 $\alpha$ deficient mice $\left(\mathrm{Mx} / \mathrm{HIF}-1 \alpha^{-}\right)$were constructed and treated with streptozotocin (STZ) injection for hypertensive DN induction. Normal C57BL/6 mice received STZ or no treatment (normal) were considered as controls. Three days post STZ administration; body weight, fasting blood glucose (FBG), 24 h urinary albumin and systolic blood pressure (SBP) were measured weekly. Periodic acid-Schiff's staining was performed for histologic analysis of glomeruli damage. In comparison with the normal control, significant upregulation and downregulation of HIF-1 $\alpha$ was, respectively, detected in diabetic and HIF-1 $\alpha^{\%}$ mice $(\mathrm{P}<0.01)$. In comparison with STZ-induced diabetic mice, HIF-1 $\alpha^{\%}+$ STZ mice displayed reduced body weight, and increased FBG, urinary albumin and SBP. PAS showed HIF-1 $\alpha^{-}+$STZ mice had damaged kidney tissues, with more renal fibrosis and apparent glomerular hypertrophy. These results demonstrated that HIF-1 $\alpha$ deficiency accelerated DN progression with increasing hypertension in mice.
\end{abstract}

\section{Introduction}

Diabetic nephropathy (DN) is a leading cause of chronic kidney disease (CKD) and a major complication of diabetes. Hypertension, proteinuria and edema are frequent clinical features of DN patients and experimental DN rats (1). Hypertension elevates blood pressure, increases the kidney damages, and thus becomes an important reason leading to diabetes, DN and end-stage renal failure (ESRF) $(1,2)$. In

Correspondence to: Dr Hui Liu, Department of Endocrinology, Luoyang Central Hospital Affiliated to Zhengzhou University, 288 Zhongzhou Road, Xigong, Luoyang, Henan 471009, P.R. China E-mail: liuhui_1@126.com

Key words: hypoxia inducible factor- $1 \alpha$, diabetic nephropathy, hypertension addition, the morbidity of hypertension in diabetic patients was $1.5 \sim 3.0$ times higher than that in non-diabetic populations, showing mutual promotion effect between hypertension and DN.

Diabetic rats always displayed increased oxygen consumption and tissue hypoxia throughout the kidney (3). Chronic hypoxia causes several public health problems including renal fibrosis, reduced renal function, DN, hypertensive nephropathy, CKD, and is the final pathway to ESRF (4-8). Chronic hypoxia-induced hypertension is associated with microvascular and tubulointerstitial injury $(9,10)$. Chronic hypoxia also plays a central pathogenic role in the process of end-stage renal disease (ESRD), including ESRF (11).

The activation of hypoxia-inducible factors (HIFs), however, could prevent the progression of DN (3). Hypoxia inducible factor- $1 \alpha(\mathrm{HIF}-1 \alpha)$ is an oxygen-sensitive gene and is a transcription factor that regulates a wide variety of genes (7). HIF-1 $\alpha$ is recognized to be the essential response regulator to hypoxia, and its expression has been evidenced to be correlated with early progression of some diseases including diabetic retinopathy which was caused by hypoxia-induced hyperglycemia (6). Some evidences show HIF-1 $\alpha$ 's activation was associated with hypertension of artery, pulmonary or elsewhere (12-14). Researches had shown that overexpression of HIF-1 $\alpha$ in tubular epithelial cells contributed to renal fibrosis progression, and inhibition of HIF- $1 \alpha$ expression prevented the progression of renal fibrosis and attenuated DN progression $(5,7,11,15)$. They found HIF-1 $\alpha$-mediated abnormal pathways or genetic dysregulations triggered hypertension (16). For instance, Luo et al showed the elevated expression of endothelial HIF-1 $\alpha$ in $\mathrm{V}$-Cadherine/HIF-1 $\alpha$ floxed mice promoted the progression of angiotensin II infusion-induced hypertensive CKD (5). On the contrary, the deletion of HIF-1 $\alpha$ was reported to attenuated chronic hypoxia induced hypertension, proteinuria, kidney damage and so on (12-14,17). Some studies showed stabilization or activation of HIF-1 $\alpha$ was essential under hypoxic conditions $(7,18)$, suggesting the important roles of HIF-1 $\alpha$ expression in hypertension. However, little is known about the roles of HIF-1 $\alpha$ deficiency in hypertensive DN progression.

The present study was designed to investigate the effect of HIF-1 $\alpha$ deficiency on DN with hypertension. Mx/HIF-1 $\alpha^{-}$ mice were constructed and used as animal HIF-1 $\alpha /$-deficient 
DN model in this study. Biochemical and physiological changes in normal or HIF-1 $\alpha$ deficient mice were detected to assess the influence of HIF-1 $\alpha$ deficiency on DN progression.

\section{Materials and methods}

Animal and rats model. Mice labeled with $\mathrm{Mx}-\mathrm{Cre}$ transgenic mice (JAXMICE: 003556, Strain name: B6.Cg-Tg (Mxl-Cre) $1 \mathrm{Cgn} / \mathrm{J}$ ) were mated to HIF-1 $\alpha$ gene LoxP mice (JAXMICE: 007561, B6.129-Hif1 $\alpha$ tm3Rsjo/J, HIF-1 $\alpha$ homozygous floxed mice contain the HIF-1 $\alpha$ exon 2 flanked by LoxP sites), both were purchased from the Jackson Laboratories (Jackson Laboratory, Ben Harbor, ME, USA). Mice were allowed twice hybridization, and the identified Mx-Cre + and HIF-1 $\alpha$ floxed mice $\left(\mathrm{Mx} / \mathrm{HIF}-1 \alpha^{-/}\right)$in the third generation were used. Mice were intraperitoneally injected with $400 \mu \mathrm{l} /$ time poly (I:C; Sigma-Aldrich; Merck KGaA, Darmstadt, Germany) every 4 days for a total of 3 times for interferon induction (19). DN induction were performed on C57BL/6 mice (12 weeks old, $n=15)$ and $M x / H I F-1 \alpha^{-}$mice $(n=15)$. All mice received intraperitoneal injection of $140 \mathrm{mg} / \mathrm{kg}$ (weight body) streptozotocin (STZ; Sigma-Aldrich; Merck KGaA). Another 15 mice received injection of equal volume normal saline and were used as blank control. Mice were maintained on a normal diet. Diabetic mice was recognized when fasting blood glucose $(\mathrm{FBG}) \geq 16.7 \mathrm{mmol} / 1$ at $72 \mathrm{~h}$ post STZ injection. Then animal body weight, $24 \mathrm{~h}$ urine albumin, blood pressure, serum glucose were measured weekly. Hypertensive model was recognized with elevated systolic blood pressure (SBP) $>140 \mathrm{mmHg}$. All animals were killed by intraperitoneally administering overdose of sodium pentobarbital $(180 \mathrm{mg} / \mathrm{kg}$ body weight) at 16 weeks (20). These studies were approved by the Institutional Animal Care and Use Committee Affiliated to Zhengzhou University (Zhengzhou, China).

Blood pressure measurement. At the time of diabetic mice model construction, mice SBP was measured once a week using noninvasive BP tail-cuff with PowerLab system (ADInstruments, Mountain View, CA, USA) as previously described $(21,22)$. Prior to experimental protocols, mice were trained for 2 weeks (twice a week). Moreover, acclimation before each recording was needed.

Biochemistry investigation. Twenty-four hour urine were collected from housed mice. Urine albumin was measured using a ELISA kit (Bethyl Laboratories, Inc., Montgomery, TX, USA). FBG in tail vein were detected weekly using a glucometer (OneTouch Ultra; Lifescan, Inc., Milpitas, CA, USA).

Histologic analysis. Sixteen weeks later, mice were killed and kidney tissues were fixed, imbedded, and $2 \mu \mathrm{m}$ sections were stained with periodic acid-Schiff's (PAS) reagent at $37^{\circ} \mathrm{C}$ for $10 \mathrm{~min}$ and hematoxylin at $37^{\circ} \mathrm{C}$ for $5 \mathrm{~min}$, followed by image analysis using Image Pro Plus (Media Cybernetics, Silver Spring, MD, USA).

Western blot analysis. Kidney tissues were lysed, prepared, and separated using 10\% SDS-PAGE. Then proteins were transblotted onto Millipore PVDF membranes (EMD Millipore, Billerica, MA, USA) which were then blocked with milk. Then membranes were subjected to incubation in the specific primary antibody anti-HIF-1 $\alpha$ (1:1,500 in dilution; Cell Signaling Technology, Inc., Danvers, MA, USA) at $4^{\circ} \mathrm{C}$ overnight, and HRP-conjugated secondary antibodies for $1 \mathrm{~h}$. GAPDH (1:2,000; Cell Signaling Technology, Inc.) was used as internal reference gene. Immunoreactive protein bands were analyzed using a Bio-Rad Quantity One software (Bio-Rad Laboratories, Inc., Hercules, CA, USA) by a chemiluminescence reaction system.

Statistical analysis. Data were recorded as means \pm SD (standard deviation). Analysis was performed using GraphPad Prism v.6.0 (GraphPad Software Inc., La Jolla, CA, USA). Statistical differences among three groups were analyzed using one-way ANOVA followed by Bonferroni's post hoc test. All datasets were considered normally distributed and analyzed using parametric statistics. $\mathrm{P}<0.05$ was considered to indicate a statistically significant difference.

\section{Results}

HIF-1 $\alpha$ knockout promotes DN pathogenesis. HIF-1 $\alpha$ protein were significantly upregulated in diabetic mice in comparison with the control mice $(\mathrm{P}<0.01$; Fig. 1). The significant downregulation of HIF-1 $\alpha$ protein (not complete knockout) in $\mathrm{Mx} / \mathrm{HIF}-1 \alpha^{-/}$mice was determined using western blot analysis, which confirmed the knockout of HIF-1 $\alpha$ in $\mathrm{Mx} / \mathrm{HIF}-1 \alpha^{\%}$ mice (Fig. 1). The little expression of HIF-1 $\alpha$ protein in $\mathrm{Mx} / \mathrm{HIF}-1 \alpha^{-/}$mice might due to the HIF-1 $\alpha$ homolog.

Effect of HIF-1a knockout on body weight, urinary albumin, blood glucose and pressure. The body weight, FBG, $24 \mathrm{~h}$ urinary albumin, and SBP in 3 groups were investigated. No death was observed in control (C57BL/6) mice, and 5 mice in STZ group died during the 16 weeks. All Mx/HIF-1 $\alpha /$ mice died in the first 9 weeks. So the investigation of HIF-1 $\alpha^{\%} /$ mice were available in the first 9 weeks.

During the experimental protocol, non-diabetic mice had the expected low level of FBG, urinary albumin, and SBP value. In comparison, $\mathrm{HIF}-1 \alpha^{-}+\mathrm{STZ}$ mice showed the lowest body weight, and the highest levels of FBG (>20 mmol/l), $24 \mathrm{~h}$ urinary albumin and SBP during the 16 weeks' experimental protocol (Fig. 2). As expected, the body weight of non-diabetic mice was gradually increased during the experiment period and was already significantly higher than mice treated with STZ at the end of first week $(\mathrm{P}<0.01$; Fig. 2A). On the contrary, the body weight of mice with diabetes (STZ and HIF-1 $\alpha^{-}+$ STZ) was declined during the experiment period. The FBG levels of the diabetic mice (STZ and HIF-1 $\alpha^{-}+\mathrm{STZ}$ ) were significantly higher than those of control mice (C57BL/6) at the beginning $(\mathrm{P}<0.001)$, and were gradually decreased since the third week, indicating the induction of diabetic mice by STZ administration. In comparison with the gradually increased urinary albumin level in HIF-1 $\alpha^{-}+$STZ mice, that of the STZ or $\mathrm{C} 57 \mathrm{BL} / 6$ mice did not increased during all the experimental protocol (Fig. 2C), revealing the development of DN in $\mathrm{HIF}-1 \alpha^{-}$ + STZ mice. No increment in urinary albumin was observed in STZ-induced diabetic mice compared with non-diabetic C57BL/6 mice ( $\mathrm{P}>0.05)$, suggesting there was no DN incidence in STZ-induced diabetic mice. At last we found the diabetic 

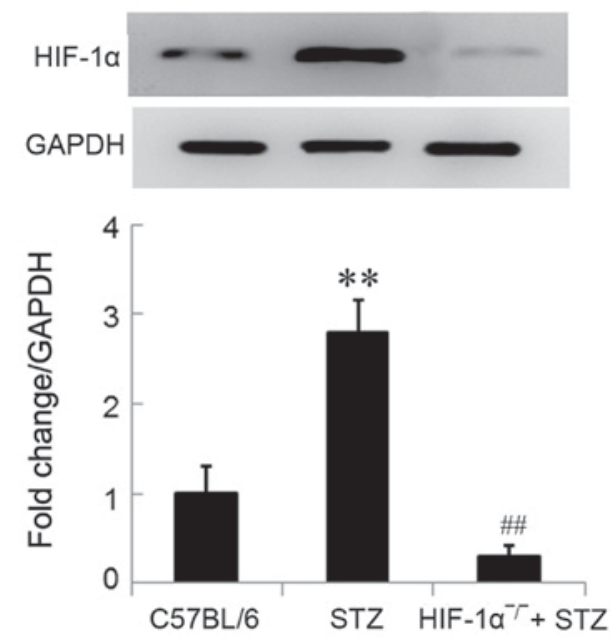

Figure 1. Expression of HIF-1 $\alpha$ protein in mice. HIF-1 $\alpha$ expression was significantly enhanced in diabetic mice (STZ) and was knocked out in HIF-1 $\alpha$-deficient $\left(\mathrm{HIF}-1 \alpha^{-} /\right)+$STZ mice. Data are expressed by mean \pm SD . ${ }^{* *} \mathrm{P}<0.01$ vs. control (C57BL/6). ${ }^{\# \#} \mathrm{P}<0.01$ vs. STZ. STZ, streptozotocin; SD, standard deviation.

mice displayed relative higher SBP values in comparison with C57BL/6 mice (non-diabetic, $\mathrm{P}<0.01$; Fig. 2D). The HIF-1 $\alpha^{\%}+$ STZ mice developed with SBP value of more than $140 \mathrm{mmHg}$ at the fifth week, indicating the development of hypertensive mice. The SBP value in STZ group was lower than $140 \mathrm{mmHg}$, revealing there was no hypertensive mice in STZ group although the SBP value was relative higher than that in control mice $(\mathrm{P}<0.05)$. Taken together, these results demonstrated that HIF-1 $\alpha$ deficiency distinctly accelerated the DN pathogenesis.

HIF-1 $\alpha$ knockout promotes glomerular damage. To determine the damage of HIF-1 $\alpha$ deficiency on kidney function, we performed the PAS staining on glomerular. We found that diabetic mice with HIF-1 $\alpha$ deficiency showed the accelerated severity of glomerular damage compared with STZ-induced diabetic mice (Fig. 3). Diabetic mice, especially HIF-1 $\alpha^{-/}$mice, showed progressive histopathologic changes in glomeruli and tubulointerstitium. HIF-1 $\alpha^{-/}$mice showed distinct renal injury histologic properties including collagen deposition in glomeruli, glomerular sclerosis, necrotic lysis, tubular brush border loss, tubular dilatation, and sloughing of cellular debris into the tubular lumen. In comparison with HIF-1 $\alpha^{-}+$STZ mice, mice in STZ group showed slight histopathologic changes. These distinct histopathologic changes in HIF-1 $\alpha \%$ diabetic mice confirmed DN and suggested that HIF-1 $\alpha$ deficiency promoted glomerular damage.

\section{Discussion}

We confirmed that DN progression was associated with downregulation of HIF-1 $\alpha$. We found the knockout of HIF-1 $\alpha$ promoted $\mathrm{DN}$ progression in mice. In comparison with STZ-induced diabetic mice, HIF-1 $\alpha$ deficient mice plus STZ showed increased FBG and SBP level, and enhanced DN pathological characters of aggravated glomeruli damage and elevated levels of $24 \mathrm{~h}$ urinary albumin. We demonstrated that HIF-1 $\alpha$ deficiency promoted the DN progression in diabetic mice.
HIF-1 $\alpha$ expression could be induced by hypoxia and was correlated with early progression of some diseases (6). One of the leading causes of DN in diabetes is renal hypoxia (23). Elevated HIF-1 $\alpha$ expression in kidney of mice with hypertensive nephropathy and renal fibrosis had been proved to promote the progression of diseases $(5,11)$. On contrast, the deletion of HIF-1 $\alpha$ could attenuate disease progression, and showing protective effect on kidney function. In the present study, we confirmed the upregulation of HIF-1 $\alpha$ in hypertensive DN kidneys of mice with renal fibrosis. These demonstrated hypoxic conditions in DN progression and the contribution to kidney damage.

Referring to the HIF-1 $\alpha$ deficiency effect, reports showed HIF-1 $\alpha$ deficiency could attenuate hypertension of artery, pulmonary, proteinuria, and kidney damage $(12-14,17)$. As reported, hypoxia-induced renal tubular epithelial cell death was prevented by HIF-1 $\alpha$-deficiency, and that was a glucose dependent manner (15). Luo et al (5), demonstrated that HIF-1 $\alpha$ deficiency attenuated glomeruli damage and renal fibrosis in angiotensin II-infused mice. Shimoda et al (17), showed an partial HIF-1 $\alpha$ deficiency of Hifla (+/-) could blunt hypoxia-induced right ventricular hypertrophy and polycythemia in vivo in PASMCs. Also, in comparison with reduced $\mathrm{K}+$ current density in PASMCs from HIF-1 $\alpha(+/+)$, no reduction in $\mathrm{K}+$ current density was observed in PASMCs of hypoxic HIF-1 $\alpha$ (+/-) mice (17). Hu et al (13), showed overexpression of HIF-1 $\alpha$ in fructose-induced salt-dependent hypertensive rats attenuated high salt + fructose-induced high blood pressure. Taken together, these results showed HIF-1 $\alpha$ deficiency benefited to attenuate hypertension or kidney damage.

In the present study, however, we demonstrated that HIF-1 $\alpha$ deficiency accelerated hypertensive DN progression in $\mathrm{Mx} / \mathrm{HIF}-1 \alpha^{-/ m i c e}$. HIF-1 $\alpha$ deficient mice showed aggravated kidney damage, higher FBG and SBP levels, and elevated collagen deposition, suggesting the progressive roles of HIF-1 $\alpha$ deficiency in STZ-induced DN. Our results was in accordance with the that from Bohuslavova et al (24), who stated the heterozygosity HIF-1 $\alpha$ knockout (HIF-1 $\alpha+/-)$ mice displayed diabetic cardiomyopathy with decreased LVFS and myocardial function. They found STZ inhibited HIF-1 $\alpha$ expression in wild type mice and upregulated HIF-1 $\alpha$ and Coll in HIF-1 $\alpha+/$-mice, suggesting the genetic variation at the HIF-1 $\alpha$ locus influenced the extracellular matrix reorganization and risk for fibrosis and diabetic cardiomyopathy. We guessed the aggravated DN in HIF-1 $\alpha$-deficient mice might due to the HIF- $1 \alpha$ deficiency-induced deletion of compensatory effect to hypoxia responses, that led to the loss of hypoxia preconditioning. Finally, elevated hypoxic responses in kidney tissues promoted the transdifferentiation of renal tubular epithelial cells to fibroblasts, and facilitated renal fibrosis and irreversible kidney damage.

In the present study, we also found that the urinary albumin in HIF-1 $\alpha$ deletion mice were significantly upregulated compared with those in WT mice administrated with STZ. This revealed that HIF-1 $\alpha$ deficiency promoted damage on glomerular filtration membrane, thus promoting the filtration of urinary albumin to blood. However, no increment in urinary albumin was found in STZ-treated WT mice compared with control. Generally, the decreased body weight and increased FBG, urinary albumin and SBP were predictors of DM (25). The elevated urinary albumin is a hallmark of diabetic-related kidney disease (26). We did not found the upregulated urinary 

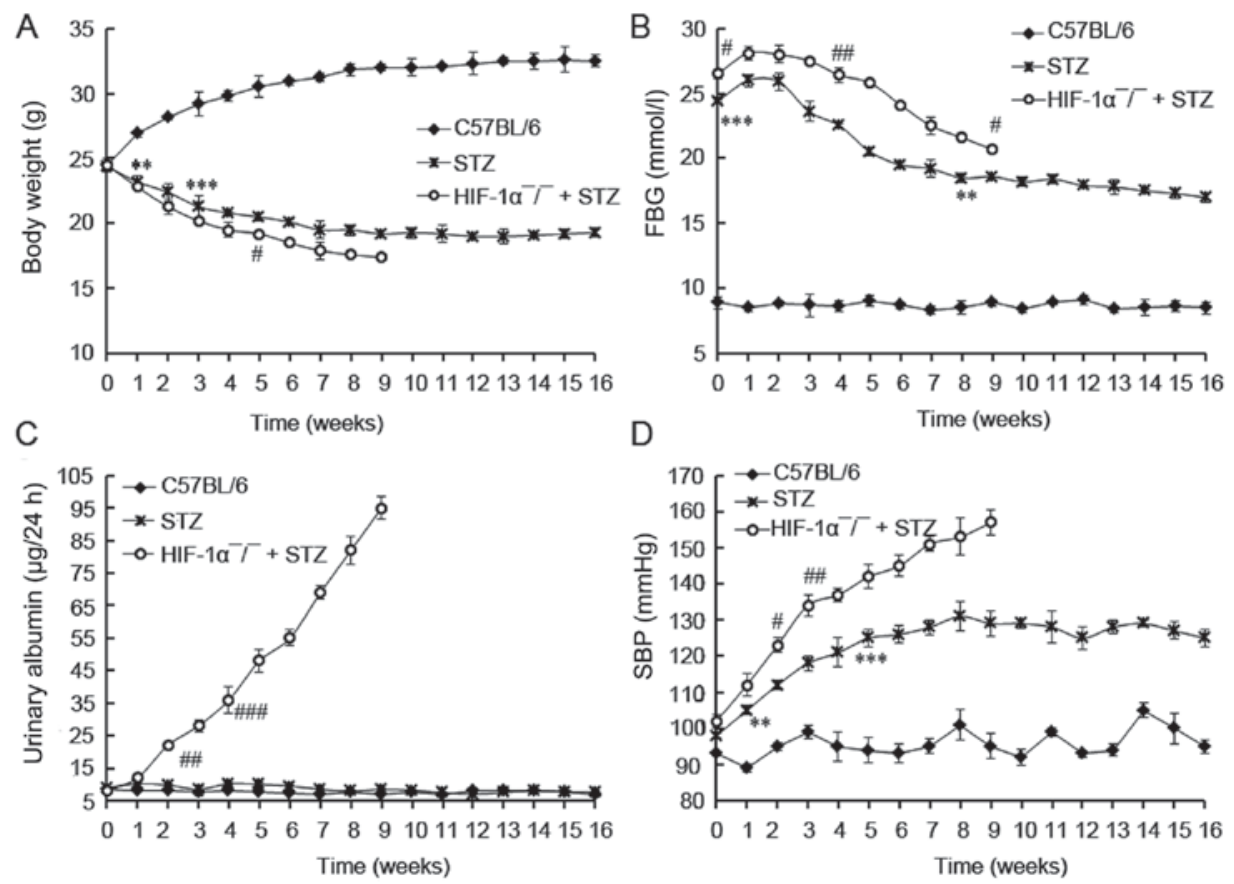

Figure 2. The investigation of biochemical parameters in diabetic rats. (A) Body weight; (B) FBG content; (C) urinary albumin content; (D) BP content. Mice were treated with STZ for induction of diabetes. The investigation of body weight, FBG, urinary albumin, and SBP in mice were started at 3 days post STZ performed weekly during 16 weeks post diabetic induction. HIF-1 $\alpha$ deficiency promotes DN progression. Data are expressed by mean \pm standard deviation

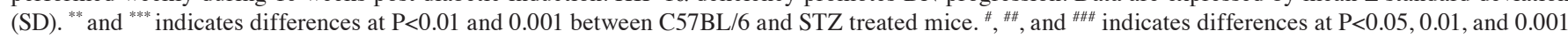
between HIF-1 $1 \gamma^{\%}+$ STZ and STZ treated mice. STZ, streptozotocin; FBG, fasting blood glucose; SBP, systolic blood pressure; DN, diabetic nephropathy.
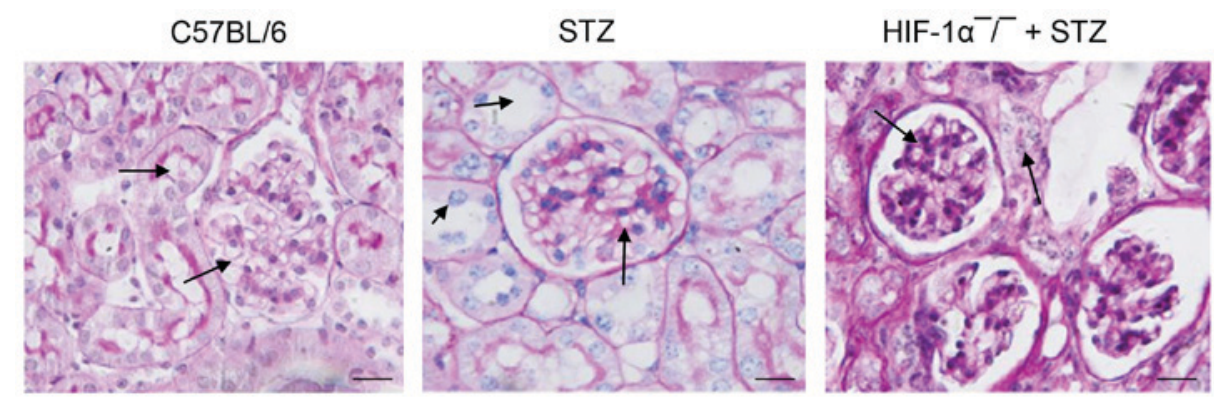

Figure 3. Histopathology analysis of kidney tissues. Kidney tissues were stained with PAS. Arrows indicate the renal fibrosis, collagen deposition, and pathology changes in glomerular. HIF-1 $\%$ mice displays increased kidney damage. Original magnification, $\mathrm{x} 300$. Scale bar=50 $\mu$ m. PAS, periodic acid-Schiff's.

albumin in mice in WT group after being administrated with STZ as HIF-1 $\alpha$ deficient mice showed, but found the slight histopathologic changes in kidney was in consistence with the unchanged urinary albumin level. This fact might suggested that STZ not definitely induced albumin increment, and also proved that HIF-1 $\alpha$ deficiency indeed promoted kidney damage.

Glucose inhibited hypoxia-induced HIF-1 $\alpha$ accumulation, and hyperglycemia regulated stability and function of HIF-1 $\alpha$ through proteasomal degradation in vivo (27). Gao et al (28), showed that hyperglycemia could inhibit HIF-1 $\alpha$ expression and attenuate the hypoxic-induced apoptosis of bovine aortic smooth muscle cells (BASMC). So, they suggested that hyperglycemia altered hypoxia-induced changes in vascular cell growth by inhibiting HIF-1 $\alpha$. HIF-1 $\alpha$ also mediates the accumulation of collagen by activation of tissue inhibitor of metalloproteinase-1 (TIMP-1) or TGF- $\beta 1 / \mathrm{Smad} 3$ signaling pathway. Fu et al (29), showed HIF-1 $\alpha$ contributed to regulation of collagen accumulation and renal fibrosis by cross talking with TGF- $\beta 1 / \mathrm{Smad} 3$ signaling pathway. To further investigate the mechanism related to HIF-1 $\alpha$ associated hypertensive DN progression, we would perform more studies to explore underlying mechanisms and signaling pathways.

In summary, we confirmed the HIF-1 $\alpha$ upregulation in DN kidney, together with the hyperglycemia and hypertensive. In addition, HIF-1 $\alpha$ deficiency did not attenuate DN damage, but inversely accelerated DN progression. We speculated that there must be differential pathways associated with HIF-1 $\alpha$ deficiency-promoted DN.

\section{Acknowledgements}

Not applicable.

\section{Funding}

No funding was received. 


\section{Availability of data and materials}

The data and materials used in this study are available upon reasonable request from the corresponding author once the paper has been published.

\section{Authors' contributions}

$\mathrm{HuL}$ and $\mathrm{YJ}$ participated in study design, data analysis and manuscript drafting. HJ, HaL and YY screened patients, collected and analyzed patient data, and revised the manuscript. $\mathrm{YZ}$ and $\mathrm{KZ}$ were involved in experiment conduction and data analysis.

\section{Ethics approval and consent to participate}

The Ethical approval was obtained from the Ethics Committee of The 1st Affiliated Hospital of Henan University of Science and Technology, and all participants signed a consent form prior to enrollment in the study.

\section{Patient consent for publication}

Not applicable.

\section{Competing interests}

All authors declare they have no competing interests.

\section{References}

1. Xie S, Lu K, Zhang Y, Song X, Tan M and Wang C: Effects of Jiangya Xiaoke prescription on TGF- $\beta 1$ in diabetic nephropathy rats with hypertension and its mechanisms. Int J Clin Exp Med 8: 5129-5136, 2015.

2. Grossman E: Should we treat prehypertension in diabetes? What are the cons? Diabetes Care 32 (Suppl 2): S280-S283, 2009.

3. Nordquist L, Friederich-Persson M, Fasching A, Liss P, Shoji K, Nangaku M, Hansell P and Palm F: Activation of hypoxia-inducible factors prevents diabetic nephropathy. J Am Soc Nephrol 26: 328-338, 2015.

4. Gu HF, Zheng X, Abu Seman N, Gu T, Botusan IR, Sunkari VG, Lokman EF, Brismar K and Catrina SB: Impact of the hypoxia-inducible factor-1 $\alpha$ (HIF1A) Pro582Ser polymorphism on diabetes nephropathy. Diabetes Care 36: 415-421, 2013.

5. Luo R, Zhang W, Zhao C, Zhang Y, Grenz A, Eltzschig HK, Tou L, Kellems RW and Xia Y: Rt-pcr profiling reveals the essential role of endothelial hif-1a in Hypertensive Nephropathy. Hypertension 62: A31, 2013.

6. Yan $\mathrm{H}$ and Su GF: Expression and significance of HIF-1 $\alpha$ and VEGF in rats with diabetic retinopathy. Asian Pac J Trop Med 7: 237-240, 2014.

7. Kimura K, Iwano M, Higgins DF, Yamaguchi Y, Nakatani K, Harada K, Kubo A, Akai Y, Rankin EB, Neilson EG, et al: Stable expression of HIF-1alpha in tubular epithelial cells promotes interstitial fibrosis. Am J Physiol Renal Physiol 295: F1023-F1029, 2008

8. Nangaku M: Chronic hypoxia and tubulointerstitial injury: A final common pathway to end-stage renal failure. J Am Soc Nephrol 17: 17-25, 2006.

9. Steudel W, Scherrer-Crosbie M, Bloch KD, Weimann J, Huang PL, Jones RC, Picard MH and Zapol WM: Sustained pulmonary hypertension and right ventricular hypertrophy after chronic hypoxia in mice with congenital deficiency of nitric oxide synthase 3. J Clin Invest 101: 2468-2477, 1998.

10. Mazzali M, Jefferson JA, Ni Z, Vaziri ND and Johnson RJ: Microvascular and tubulointerstitial injury associated with chronic hypoxia-induced hypertension. Kidney Int 63: 2088-2093, 2003.
11. Fu W, Wang Y, Jin Z, Wang H, Cheng W, Zhou H, Yin P and Peng W: Losartan alleviates renal fibrosis by down-regulating HIF-1 $\alpha$ and up-regulating MMP-9/TIMP-1 in rats with 5/6 nephrectomy. Ren Fail 34: 1297-1304, 2012.

12. Yamaji-Kegan K, Takimoto E, Semenza GL and Johns RA: Hypoxia-inducible factor $1 \alpha$ is a critical downstream mediator for hypoxia-induced mitogenic factor (FIZZ1/RELM $\alpha$ )-mediated pulmonary hypertension, in A51. Experimental models of pulmonary hypertension. Am J Respir Crit Care Med 191: A1924, 2015.

13. Hu J,Zhu Q, Li PL, Boini KM and Li N: Inhibition of hypoxia inducible factor- $1 \alpha$ in the renal medulla contributes to fructose-induced salt-sensitive hypertension. FASEB J 30 (1 Suppl): 1216.2, 2016.

14. Veith C, Zakrzewicz D, Dahal BK, Bálint Z, Murmann K, Wygrecka M, Seeger W, Schermuly RT, Weissmann N and Kwapiszewska G: Hypoxia-or PDGF-BB-dependent paxillin tyrosine phosphorylation in pulmonary hypertension is reversed by HIF-1 $\alpha$ depletion or imatinib treatment. Thromb Haemost 112: 1288-1303, 2014

15. Biju MP, Akai Y, Shrimanker N and Haase VH: Protection of HIF-1-deficient primary renal tubular epithelial cells from hypoxia-induced cell death is glucose dependent. Am J Physiol Renal Physiol 289: F1217-F1226, 2005.

16. Bonnet S, Michelakis ED, Porter CJ, Andrade-Navarro MA, Thébaud B, Bonnet S, Haromy A, Harry G, Moudgil R, McMurtry MS, et al: An abnormal mitochondrial-hypoxia inducible factor-1alpha-Kv channel pathway disrupts oxygen sensing and triggers pulmonary arterial hypertension in fawn hooded rats: Uimilarities to human pulmonary arterial hypertension. Circulation 113: 2630-2641, 2006.

17. Shimoda LA, Manalo DJ, Sham JS, Semenza GL and Sylvester JT: Partial HIF-1alpha deficiency impairs pulmonary arterial myocyte electrophysiological responses to hypoxia. Am J Physiol Lung Cell Mol Physiol 281: L202-L208, 2001.

18. Basu RK, Hubchak S, Hayashida T, Runyan CE, Schumacker PT and Schnaper HW: Interdependence of HIF- $1 \alpha$ and TGF- $\beta /$ Smad3 signaling in normoxic and hypoxic renal epithelial cell collagen expression. Am J Physiol Renal Physiol 300: F898-F905, 2011.

19. Du G, Leone M, Romeijn S, Kersten G, Jiskoot W, Bouwstra JA: Immunogenicity of diphtheria toxoid and poly(I:C) loaded cationic liposomes after hollow microneedle-mediated intradermal injection in mice. Int J Pharm 547: 250-257, 2018.

20. Zhu H, Li X, Yuan M, Wan W, Hu M, Wang X and Jiang X: Intramyocardial delivery of bFGF with a biodegradable and thermosensitive hydrogel improves angiogenesis and cardio-protection in infarcted myocardium. Exp Ther Med 14: 3609-3615, 2017.

21. Lim AK, Ma FY, Nikolic-Paterson DJ, Ozols E, Young MJ, Bennett BL, Friedman GC and Tesch GH: Evaluation of JNK blockade as an early intervention treatment for type 1 diabetic nephropathy in hypertensive rats. Am J Nephrol 34: 337-346, 2011.

22. Jara A, Benner CM, Sim D, Liu X, List EO, Householder LA, Berryman DE and Kopchick JJ: Elevated systolic blood pressure in male GH transgenic mice is age dependent. Endocrinology 155: 975-986, 2014

23. Franzén $S$ : The role of hypoxia for the development of diabetic nephropathy: Temporal relationship and involvement of endothelin receptor signaling. Linköping Univ Electron Press, pp53, 2016.

24. Bohuslavova R, Kolar F, Sedmera D, Skvorova L, Papousek F, Neckar J and Pavlinkova G: Partial deficiency of HIF-1 $\alpha$ stimulates pathological cardiac changes in streptozotocin-induced diabetic mice. BMC Endocr Disord 14: 11, 2014.

25. Mori KP, Yokoi H, Kasahara M, Imamaki H, Ishii A, Kuwabara T, Koga K, Kato Y, Toda N, Ohno S, et al: Increase of total nephron albumin filtration and reabsorption in diabetic nephropathy. J Am Soc Nephrol 28: 278-289, 2017.

26. Teumer A, Tin A, Sorice R, Gorski M, Yeo NC, Chu AY, Li M, Li Y, Mijatovic V, Ko YA, et al: Genome-wide association studies identify genetic loci associated with albuminuria in diabetes. Diabetes 65: 803-817, 2016.

27. Catrina SB, Okamoto K, Pereira T, Brismar K and Poellinger L: Hyperglycemia regulates hypoxia-inducible factor-1alpha protein stability and function. Diabetes 53: 3226-3232, 2004.

28. Gao W, Ferguson G, Connell P, Walshe T, Murphy R, Birney YA, O'Brien C and Cahill PA: High glucose concentrations alter hypoxia-induced control of vascular smooth muscle cell growth via a HIF-1alpha-dependent pathway. J Mol Cell Cardiol 42: 609-619, 2007.

29. Fu W, Wang Y, Jin Z, Wang H, Cheng W, Zhou H, Yin P and Peng W: Losartan alleviates renal fibrosis by down-regulating HIF- $1 \alpha$ and up-regulating MMP-9/TIMP-1 in rats with 5/6 nephrectomy. Ren Fail 34: 1297-1304, 2012. 